\title{
Mental Health Care Delivered to Younger and Older Adults by Office-Based Physicians Nationally
}

\author{
Donovan T. Maust, MD, MS, ${ }^{* \neq}$ Helen C. Kales, MD, ${ }^{* \neq}$ and Frederic C. Blow, PhD $* \%$
}

OBJECTIVES: To compare the provision of mental health care to older adults with that provided to younger adults.

DESIGN: Analysis of the National Ambulatory Medical Care Survey.

SETTING: Visits to office-based physicians in the United States, 2007 to 2010 ( $\mathrm{n}=100,661$ visits).

PARTICIPANTS: Individuals with an outpatient visit resulting in a mental health diagnosis or treatment, defined as a visit resulting in a mental disorder diagnosis, at which a psychotropic medication was prescribed, to a psychiatrist, or including psychotherapy.

MEASUREMENTS: The number of each type of mental healthcare visit was estimated according to participant age ( 21 to $64, \geq 65$ ), along with the percentage this represented of all office-based care and the annual visit rate per 100 population. Within each visit type, age groups were compared according to clinical and demographic characteristics such as sex, diagnosed mental illness, and use of psychotropic agents.

RESULTS: Older adults had a smaller proportion than younger adults of visits with a mental disorder diagnosis $(4.8 \%$ vs $9.5 \%$, chi-square $=228.21, P<.001)$, to a psychiatrist $(0.9 \%$ vs $4.0 \%$, chi-square $=233.76, P<.001)$, and including psychotherapy $(0.6 \%$ vs $2.3 \%$, chisquare $=57.65, P<.001)$. The percentage of older adult psychotropic visits was slightly smaller than of younger adult visits $(18.1 \%$ vs $19.2 \%$, chi-square $=5.33, P=.02)$. Older adults had a higher rate of psychotropic visits (121.4 per 100 population) than younger adults (56.8 per 100 population).

CONCLUSION: Less care of older adults is from psychiatrists or incorporates psychotherapy. Older adults have a far higher rate of psychotropic use than younger adults on a per-population basis. Addressing the mental healthcare

From the *Department of Psychiatry, University of Michigan; ${ }^{\dagger}$ Institute for Healthcare Policy and Innovation, University of Michigan; and ${ }^{\ddagger}$ Center for Clinical Management Research, Veterans Affairs Ann Arbor

Healthcare System, Ann Arbor, Michigan.

Address correspondence to Donovan T. Maust, Department of Psychiatry, NCRC, 2800 Plymouth Road, Building 16, Room 222W, University of Michigan, Ann Arbor, MI 48109. E-mail: maustd@umich.edu

DOI: $10.1111 /$ jgs.13494 needs of older adults will require care in nonspecialty settings. J Am Geriatr Soc 63:1364-1372, 2015.

Key words: mental health; psychotropic; psychotherapy

$\mathbf{I}_{\mathrm{t}}^{\mathrm{n}}$ 2012, the year after the first baby boomers turned 65 , the Institute of Medicine released its report The Mental Health and Substance Use Workforce for Older Adults: In Whose Hands? ${ }^{1}$ This report highlights the lack of providers with expertise necessary to address the mental health needs of older adults, especially given the burden of mental disorders in this rapidly expanding group. ${ }^{2,3}$ Efforts to meet the challenge of delivering mental health care to older adults should arise from knowledge about the nature and extent of care they currently receive. Care of mental disorders in children relative to the adult population, for example, has received a significant amount of coverage in the academic and lay press, frequently highlighting the shortage of specialty mental health providers and increased use of psychotropic medication, ${ }^{4-7}$ but with few exceptions, ${ }^{8-10}$ prior analyses of mental health care using nationally representative data rarely study older adults separately from the general adult population.

For a variety of reasons, delivery of mental health care to older adults merits additional attention. Because of accumulation of medical comorbidity ${ }^{11}$ as people age and changes in drug metabolism, ${ }^{12,13}$ potential drug-disease or drug-drug interactions that are less likely in younger adults can complicate pharmacotherapy of mental disorders. The current version of the American Geriatrics Society Beers Criteria lists nearly every psychotropic medication as potentially inappropriate. ${ }^{14}$ Older adults' treatment preferences may be different from those of younger adults, ${ }^{15}$ and they are less likely to receive treatment in specialty mental health settings. ${ }^{8,10}$ Provider concern about older adults' cognition and ability to participate in psychotherapy may mean fewer referrals than for younger individuals, although evidence suggests that psychotherapy is feasible and possibly beneficial even for individuals with dementia. ${ }^{16,17}$ In addition, although most older adults 
ultimately have insurance coverage through Medicare, psychiatrists are less likely than other providers to accept Medicare ${ }^{18}$ and older adults may have limited income and thus be unable or unwilling to pay out of pocket, creating an additional potential barrier to receiving specialty care.

For purposes of providing care and training providers, it is important to investigate whether the current understanding of outpatient mental health treatment applies to older adults. With just 1,700 geriatric psychiatrists in practice, ${ }^{19}$ access to specialty care will be the exception rather than the norm as the older adult population continues to grow. Refining knowledge regarding where older adults with mental disorders are seen and the nature of the mental health care they receive is critical to understanding quality and improving delivery. The goal of the current study was to describe and possibly differentiate the outpatient mental health care of younger and older adults by building upon recent work. ${ }^{20}$ Four broad categories of care were examined: outpatient visits with a mental health diagnosis, visits at which a psychotropic medication was prescribed, visits to a psychiatrist, and visits including psychotherapy. What proportion of ambulatory care nationwide is dedicated to these types of visits, whether these visit types differ according to age group, and what demographic and other clinical characteristics are associated with mental health care are described.

\section{METHODS}

\section{Source of the Data}

Data for these analyses were obtained from the National Ambulatory Medical Care Survey (NAMCS), a national survey administered by the National Center for Health Statistics (NCHS; Hyattsville, MD) of the Centers for Disease Control and Prevention designed to "provide objective, reliable information about the provision and use of ambulatory medical care services in the United States." This study uses data from the 2007 to 2010 survey years, the most recent available.

\section{Survey Design}

NAMCS is a national probability sample survey of officeand community health center-based physicians that yielded 125,029 encounters over the 4 study years. Physicians are sampled from the master files that the American Medical Association and American Osteopathic Association maintain; physicians in the specialties of anesthesiology, pathology, and radiology are excluded. In addition, encounters such as house calls or those to institutional settings (e.g., nursing homes) are not included. Each physician is assigned to a 1-week reporting period, during which time office staff maintain a log of all visits during the reporting period. At the end of the week, visits are randomly selected from this list using a random start and predetermined sampling interval. The physician or office staff record data for selected visits on a standardized one-page form. The overall physician response rate over the 4 years ranged from $58.3 \%$ to $62.1 \%$. Adjusting for survey design elements allows analyses to represent total annual visits to U.S. office-based physicians. ${ }^{21}$

\section{Mental Healthcare Visit Classification}

To explore how the burden of mental disorders and rates of mental health treatment in outpatient care vary according to age, four types of visits were considered, following a recent analysis: ${ }^{20}$ visits resulting in a mental disorder diagnosis, visits at which a psychotropic medication was prescribed, visits to a psychiatrist, and visits at which psychotherapy was provided. Mental healthcare visit is used herein to refer to these four visit types considered together. For the purposes of these analyses, a mental healthcare visit does not refer exclusively to a specialty mental healthcare visit.

As part of the information collected at each NAMCS encounter, up to three diagnoses are recorded using the International Classification of Diseases, Ninth Revision, Clinical Modification and the Diagnostic and Statistical Manual of Mental Disorders, Fourth Edition. For these analyses, encounters were then classified as to the presence or absence of a given condition. ${ }^{22,23}$ For example, visit diagnosis codes of $296.2 \mathrm{x}-296.3 \mathrm{x}, 296.82,298.0 \mathrm{x}$, $300.4 \mathrm{x}, 309.1 \mathrm{x}$, and 311.x defined encounters for a depressive disorder. Similar criteria were applied to define visits for psychotic disorders (295.x, 297.x, 298.1x298.9x, 299.1x), bipolar disorder (296.0x-296.1x, 296.4x-296.81, 296.89, 301.13), anxiety disorders (300.0x, 300.2x, 300.3x, 309.21, 309.81, 313.0x), substance-related disorders (291.x-292.x, 303.x-305.x), and dementia (290.1 to $290.4,294.1,331.0,331.1,331.2$, 331.82). Last, a category was created for encounters with any psychiatric diagnosis including dementia (290 to 319, 331.0, 331.1, 331.2, 331.82).

Survey data included up to eight medications prescribed, ordered, supplied, administered, or continued during each visit. For these analyses, a visit was classified as a at which a psychotropic medication was prescribed if any of the following classes of medications (Appendix 1) was included in the visit medication list: antidepressants; anxiolytics, sedatives, and hypnotics (including benzodiazepines and nonbenzodiazepine sedatives), hereinafter referred to as anxiolytics; antipsychotics; mood stabilizers (including lithium, carbamazepine, valproate and derivatives, lamotrigine); and stimulants (including atomoxetine). A visit could include medications from multiple classes.

NAMCS classifies providers into 15 specialty groups, which for these analyses were grouped into three categories: psychiatrist, primary care provider (pediatrics, family medicine, internal medicine), or other medical specialty. The survey elicits information regarding the type of services and procedures performed during the office encounter, including a specific item (yes/no) regarding provision of psychotherapy.

After determining which surveyed visits belonged to each of the mental health visit categories, the visits were then stratified according to participant age into two categories: adult (21 to 64$)$ and older adult $(\geq 65)$. The visit categories were not mutually exclusive. For example, a visit to a general internist for a 55 -year-old individual with diagnoses of diabetes mellitus and major depressive disorder who received psychotherapy and was prescribed sertraline would be included in three of the four mental health 
care visit categories (all except psychiatrist visit) for the adult group.

\section{Other Characteristics}

Sociodemographic items from the survey used for these analyses included sex, race and ethnicity (non-Hispanic white, non-Hispanic black, Hispanic, other), payer (Medicare, Medicaid, private insurance, other; categories not mutually exclusive), urban or rural office location (determined according to Metropolitan Statistical Area status), and region of the country (northeast, south, central, west).

\section{Statistical Methods}

For each of the four mental healthcare visit types, annual estimates of office-based visits were determined, and the estimates for each type were summed over the 4 study years (2007 to 2010) and then stratified according to participant age (younger vs older adult). Analyses were adjusted for visit weight, clustering within physician practice, and stratification using survey design elements provided by NCHS to generate national visit estimates and corresponding 95\% confidence intervals (CIs). ${ }^{21}$ Survey years were combined as recommended by NCHS to produce a more-reliable annual visit rate estimate. ${ }^{22}$ To generate population-based visit rates for the four visit types, the denominator (U.S. population for age group) was obtained from U.S. Census population estimates for the 4-year study interval to derive an annual visit rate per 100 population for each age group. ${ }^{23}$ Population-based visit rates were also determined for individual psychotropic class.

After determining the population-based visit rates, subsequent analyses compared how clinical and demographic characteristics varied according to age group for each type of mental healthcare visit. Categorical variables (e.g., sex) were examined by comparing the proportion of visits in each age group that corresponded to a given category (e.g., $32.3 \%$ of older adult visits resulting in a mental disorder diagnoses were by men, vs $38.8 \%$ of adult visits). Chi-square tests were used to determine associations between demographic and clinical characteristics and the four visit types for each age group, with results weighted to allow national inferences. Analyses were conducted in Stata 13.1 (Stata Corp., College Station, TX) using twosided analyses with $\alpha=.05$. For population-based visit rates, which combine NAMCS survey data (numerator) with U.S. Census information (denominator), statistical significance was approximated according to nonoverlap of $95 \%$ CIs. $^{24}$

\section{RESULTS}

There were 125,029 office visits over the 4 survey years. When limited to younger and older adults, there were 100,661 visits. Table 1 presents the proportion of total outpatient visits for each age group in each of the four mental healthcare visit categories. Mental healthcare visits were a smaller proportion of the care of older adults than of younger adults. Of the visit types, the proportion of psychotropic visits between age groups was most comparable, although slightly lower for older adults $(18.1 \%, 95 \%$ $\mathrm{CI}=16.9$ to $19.2 \%$ vs $19.2 \%, 95 \% \mathrm{CI}=18.3$ to $20.2 \%$, chi-square $=5.33, P=.02$ ). The annual population-based visit rates for each type of mental health visit are presented in Figure 1 according to age group. The annual psychotropic visit rate (per 100 population) for older adults was $121.4(95 \% \mathrm{CI}=106.7$ to 136.1$)$, much higher than the adult rate of $56.8(95 \% \mathrm{CI}=50.6$ to 62.9$)$. Older adults had lower rates of psychiatrist and psychotherapy visits.

\section{Mental Disorder Visits}

Older women $(67.7 \%)$ were more likely than younger women $(61.2 \%)$ to have a visit with a mental disorder diagnosis (chi-square ${ }^{2}=12.86, P<.001$ ), but the proportion of these visits did not vary between age groups according to race and ethnicity, geographic area, or metropolitan status (Table 2).

A larger proportion of mental disorder visits for older adults were to primary care and other specialty providers, with $19.4 \%$ of these visits to a psychiatrist for older adults, versus $40.1 \%$ for younger adults (chi-square $=$ 81.33, $P<.001$ ). Older adults had a larger proportion of visits with a dementia diagnosis, and other diagnoses accounted for a smaller share of visits than for younger adults. For mental disorder visits, nearly $75 \%$ of participants received psychotropic medication, which did not vary according to age group.

\section{Psychotropic Visits}

For visits at which a psychotropic medication was prescribed, sex distribution did not differ between age groups. Older non-Hispanic black adults accounted for a smaller share of psychotropic visits than their younger adult

Table 1. Mental Healthcare Visits as a Percentage of Total Office-Based Physician Visits for Younger and Older Adults in the United States from 2007 to 2010

\begin{tabular}{|c|c|c|c|c|}
\hline Visit Type & $\begin{array}{l}\text { Younger Adults (21-64), } \\
n=67,653\end{array}$ & $\begin{array}{l}\text { Older Adults ( } \geq 65) \\
\qquad n=33,008\end{array}$ & Chi-Square & $P$-Value \\
\hline Total office-based visits, $n$ & $2,110.280,000$ & $1,052.840,000$ & & \\
\hline \multicolumn{5}{|c|}{ Mental health visit, \%, (95\% confidence interval) } \\
\hline Mental disorder diagnosis & $9.5(8.9-10.2)$ & $4.8(4.3-5.2)$ & 228.21 & $<.001$ \\
\hline Psychotropic medication & $19.2(18.3-20.2)$ & $18.1(16.9-19.2)$ & 5.33 & .02 \\
\hline Visit to psychiatrist & $4.0(3.5-4.5)$ & $0.9(0.75-1.2)$ & 233.76 & $<.001$ \\
\hline Psychotherapy & $2.3(1.9-2.7)$ & $0.6(0.49-0.84)$ & 57.65 & $<.001$ \\
\hline
\end{tabular}




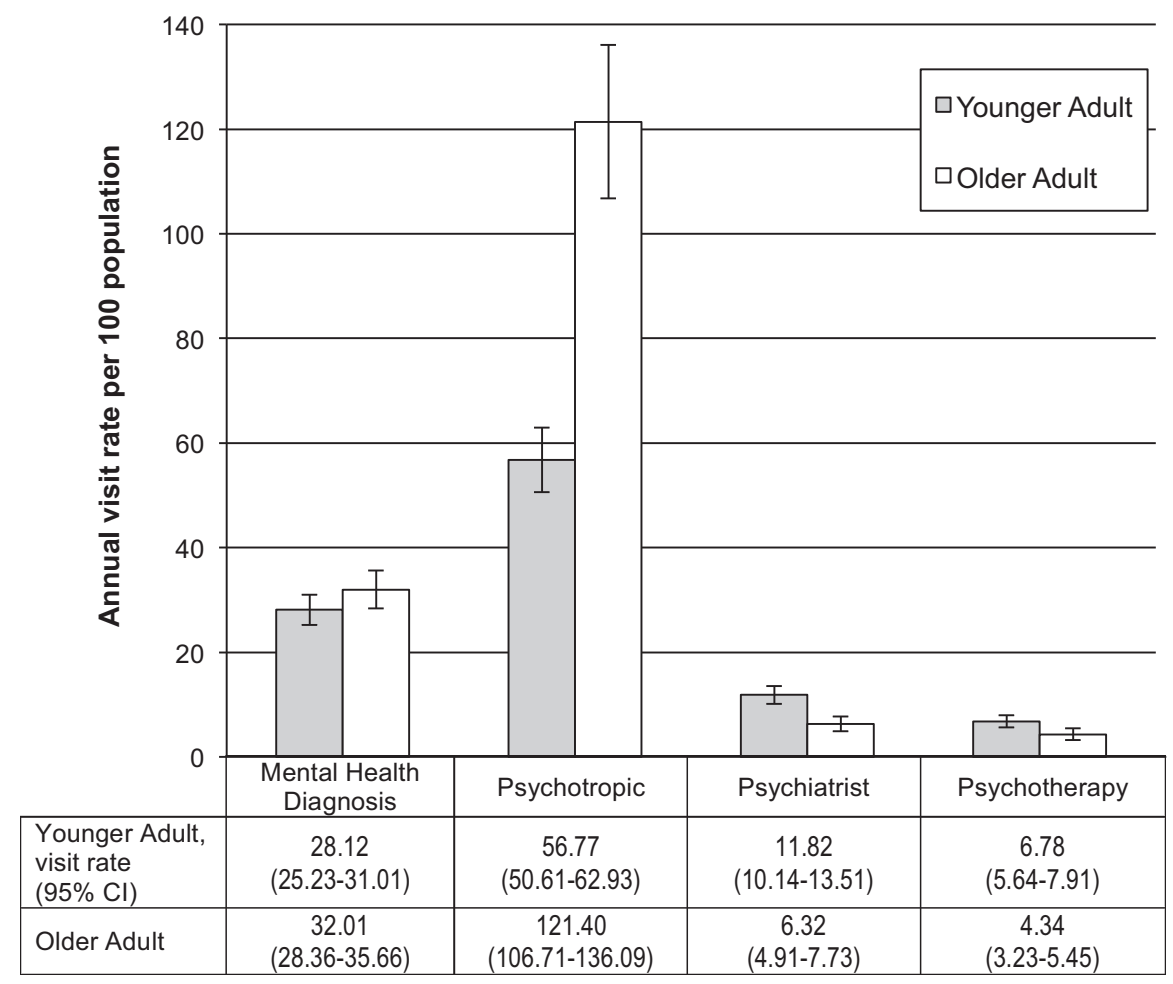

Figure 1. Annual population-based rates and $95 \%$ confidence intervals according to age group for visits with a mental disorder diagnosis, with a psychotropic medication prescription, to a psychiatrist, or for psychotherapy.

counterparts did $(6.7 \%$ vs $8.9 \%$, chi-square $=10.65$, $P=.001)$. Otherwise, psychotropic visits did not vary across age groups according to race and ethnicity or geography.

For older adult visits with a psychotropic medication prescribed, just $4.4 \%$ of visits were to a psychiatrist, compared with $17.1 \%$ for younger adults (chi-square $=175.13$, $P<.001$ ). Older adults had a mental health diagnosis at $19.3 \%$ of psychotropic visits, versus $36.8 \%$ for younger adults $($ chi-square $=191.16, P<.001)$. Depression and anxiety were the most common diagnoses at psychotropic visits for both age groups. For both age groups, approximately half of the psychotropic visits involved anxiolytics $(50.3 \%$ for younger adults, $50.8 \%$ for older adults; chisquare $=0.22, P=.64$ ). Figure 2 presents medication visits on a per capita basis according to medication type. Older adults had nearly double the rates of antidepressant and anxiolytic visits, with rates of mood stabilizer and antipsychotic use similar to those of younger adults.

\section{Visits to Psychiatrists}

For older adults, women accounted for $67.8 \%$ of psychiatrist visits, an even larger percentage than for younger adults $(60.1 \%$, chi-square $=4.67, P=.03)$, whereas older non-Hispanic black adults accounted for fewer visits than their younger counterparts $(3.3 \%$ vs $8.8 \%$, chisquare $=12.38, P<.001)$. Psychiatrist visits were the only type of mental health visit with a difference according to geographic region; a larger percentage occurred in the northeast for older adults than younger $(38.8 \%$ vs $29.3 \%$, chi-square $=4.21, P=.04$ ).

Older adults seeing a psychiatrist were more likely to have depression and dementia than younger adults and less likely to have bipolar disorder, substance use, or an anxiety disorder than younger adults.

\section{Psychotherapy Visits}

The sex balance of psychotherapy visits was not statistically significantly different between age groups. Older nonHispanic white adults accounted for a larger percentage of psychotherapy visits than younger non-Hispanic white adults $(89.4 \%$ vs $83.2 \%$, chi-square $=4.18, P=.04)$.

For older adults, primary care providers provided $20.3 \%$ of the psychotherapy visits, double the $9.6 \%$ of such visits to younger adults (chi-square $=4.42, P=.03$ ). The majority of older adult psychotherapy visits were for a depression diagnosis $(54.2 \%)$ than of younger adult psychotherapy visits $(44.8 \%) \quad($ chi-square $=4.03, P=.04)$, with a smaller share of visits than for younger adults for substance use or anxiety disorders.

\section{DISCUSSION}

Prior work has described psychotropic use and psychotherapy in general practice and specialty psychiatric settings, although these analyses generally consider the adult population overall or compare youth with adults. ${ }^{25-28} \mathrm{~A}$ recent, broad overview of office-based mental health treatment considered children, adolescents, and adults; again, treatment of older adults was not considered separately. ${ }^{20}$ Treatment of older adults with mental disorders merits additional specific attention, because they often have more comorbid medical problems, are sensitive to psychotropic medication side effects, have greater potential for polypharmacy-related adverse events, and will be the fastest 


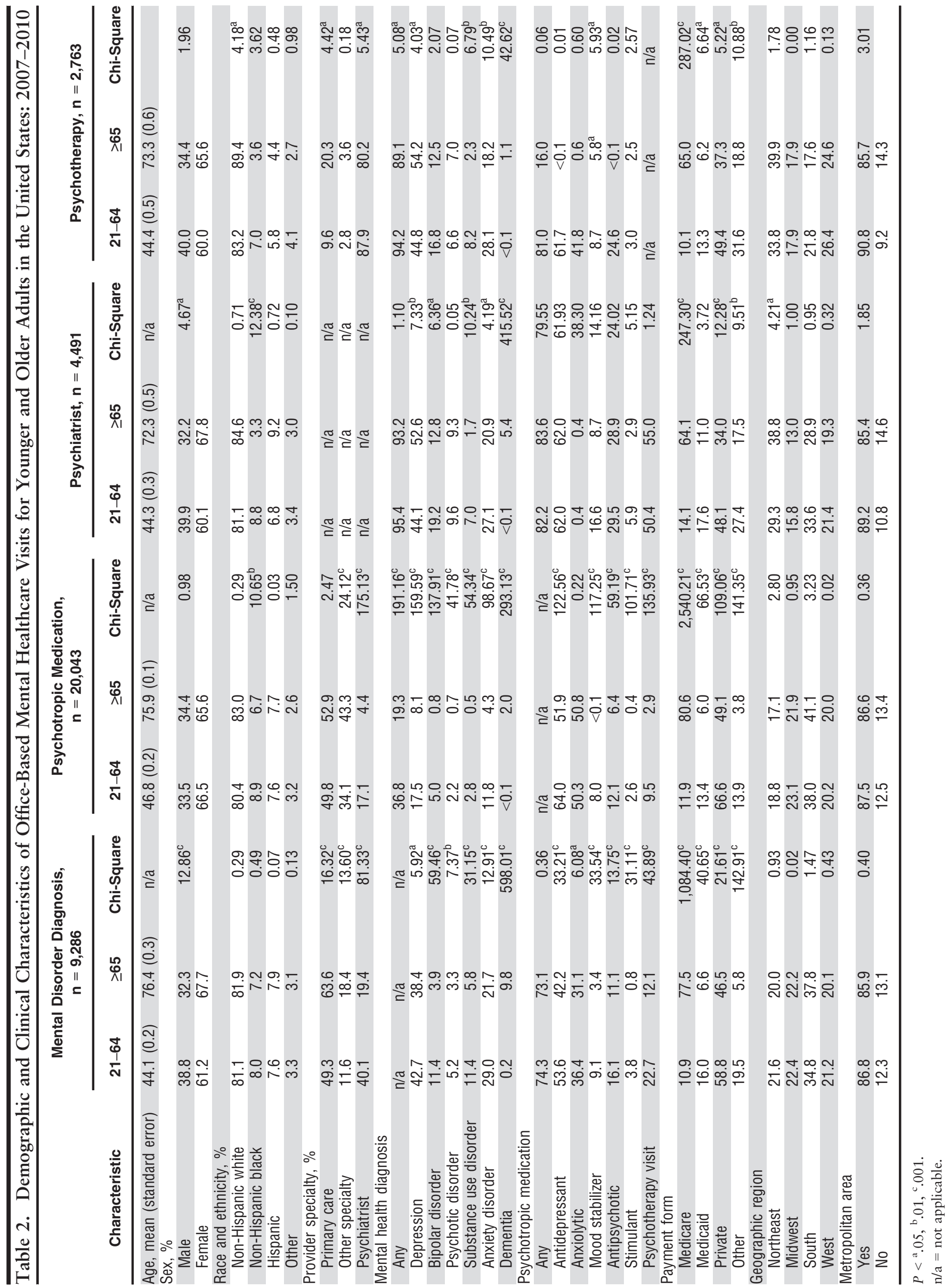




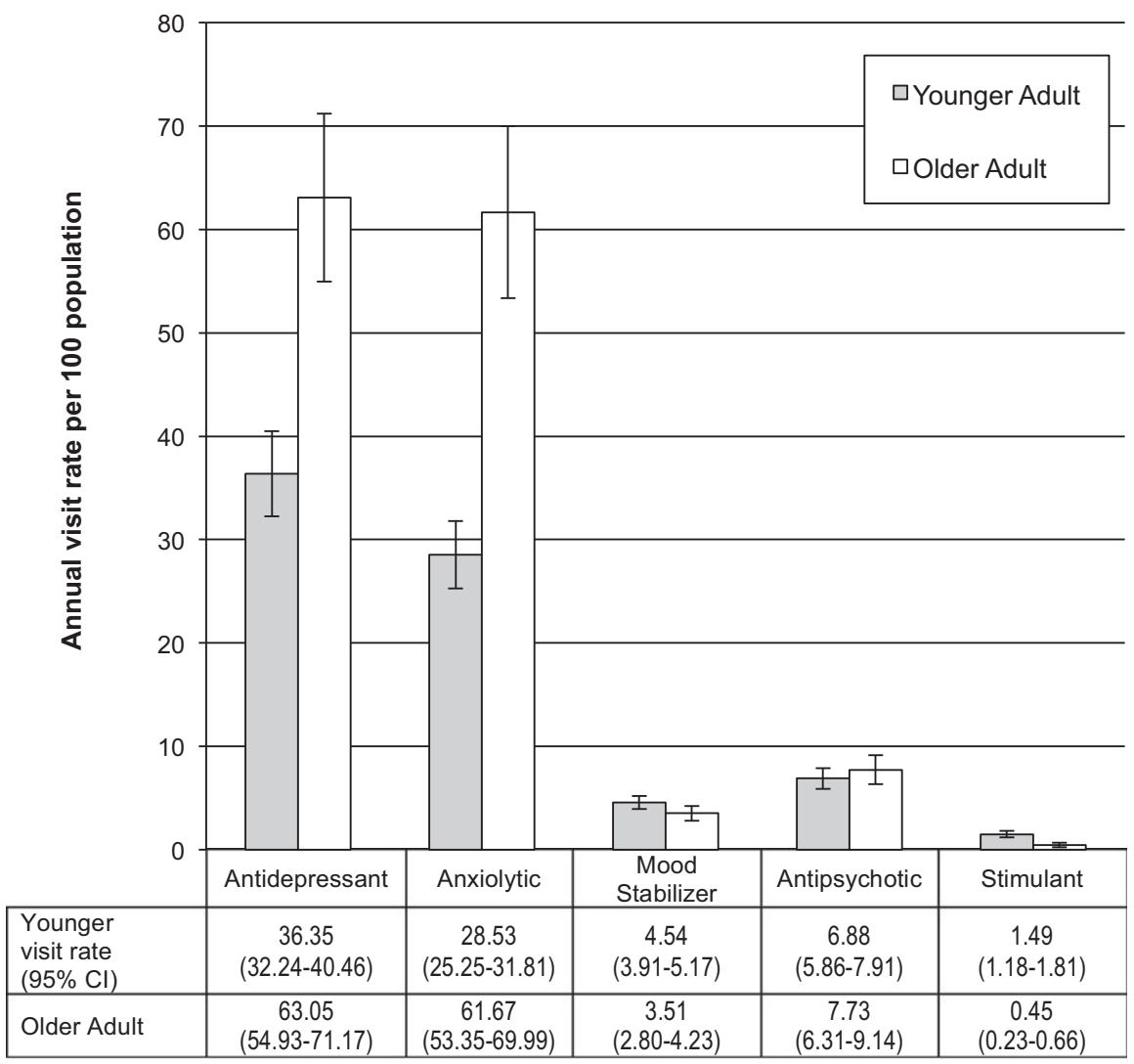

Figure 2. Annual population-based visit rates and $95 \%$ confidence intervals according to age group for psychotropic visits according to medication class.

growing segment of the population over the coming decades. ${ }^{11,29-32}$ These specific treatment-related concerns can only be addressed when paired with knowledge regarding where and what manner of treatment currently occurs.

To the knowledge of the authors of the current study, these are the first analyses to broadly consider whether and how the provision of outpatient mental health care varies between younger and older adults as a proportion of all care provided and on a per capita basis. Visits resulting in a mental disorder diagnosis accounted for $4.8 \%$ of all older adult office-based visits, approximately half the $9.5 \%$ for younger adults. On a per capita basis, the number of visits with a mental disorder diagnosis was equivalent between the two age groups, although the rate of psychiatrist and psychotherapy visits for older adults was lower, and psychotropic use was far higher. The older adult psychotropic visit rate of 121.4 found in the current study is nearly double the 65.9 reported previously as the adult population rate in an analysis of the same data. ${ }^{20}$ The high rate of anxiolytic visits is particularly troubling in light of recent evidence associating benzodiazepine use with development of dementia. ${ }^{33,34}$

This work highlights the large role of nonpsychiatrist providers in the provision of mental health care in general and for older adults in particular. The findings suggest that defining mental health care as encounters with a psychiatrist or other mental health professional does not accurately reflect where people with mental disorder diagnoses are seen and receive pharmacotherapy or psychotherapy. Fewer than $1 \%$ of all ambulatory physician encounters for older adults are to psychiatrists, and psychiatrists account for just $4.4 \%$ of visits including psychotropic use by older adults. Primary care providers account for $20.3 \%$ of older adult psychotherapy visits, in comparison with $9.6 \%$ of such visits for younger adults. It is encouraging that primary care providers are employing psychotherapy, although this may suggest a lack of access to psychiatrists because of provider shortage or psychiatrists' significantly lower rate of accepting Medicare. ${ }^{18}$ Unfortunately, the survey does not provide information about the nature of psychotherapy provided.

Considering the clinical and demographic characteristics of four visit categories, several trends emerge. First, the sex imbalance for older adults with mental disorder diagnoses and treatment is generally consistent with, if not worse than, that found in the younger adult population. Older women accounted for a larger share of visits with a mental disorder diagnosis and to a psychiatrist than younger women. Although men have a shorter life expectancy, longevity differences alone cannot account for the sex gap in mental health diagnoses and treatments in older adults. Older non-Hispanic black adults accounted for a smaller share of psychotropic visits and visits to psychiatrists than their younger counterparts, whereas older non-Hispanic white adults accounted for more psychotherapy visits. The rate of visits with mental disorder diagnoses between age groups did not vary according to race and ethnicity. The lower use of psychotropic medication may reflect treatment preferences of non-Hispanic black individuals, ${ }^{35}$ although the finding that older nonwhite adult did not 
account for more psychotherapy visits than their younger counterparts my suggest difficulty accessing this type of treatment.

This work has several limitations. Because NAMCS is a nationally representative survey of outpatient visits, individual-level clinical assessments are not available. It may be that the high rate of psychotropic prescribing for older adults is clinically appropriate and that the lower rates for adults and youth represent undertreatment, although without clinical assessments, such conclusions cannot be made. Visit diagnoses are limited to three, which means that mental disorders may be underreported (e.g., generalized anxiety disorder is item 4 on the problem list and is left off the survey instrument). This underreporting would be more likely for older adults as they have more medical conditions than adults or youth, ${ }^{11}$ so it is likely that the visit estimate for mental disorders in older adults in particular is an underestimate. In addition, it is possible that some portion of the psychotropic medication use was for a nonpsychiatric indication such as pain or insomnia, although given the lack of clinical assessment for these individuals and the limited list of diagnoses, it is difficult to make inferences about potentially off-label use with this data source.

For the first time, these findings present data that allow direct comparison for older adults relative to younger adults for office-based mental health visits nationally. As such, the findings suggest that patterns of care for older adults are distinct from those for younger adults. Although visits with a mental disorder diagnosis for older adults account for approximately half the proportion of such visits for younger adults, psychotropic use is similar, whereas psychiatrist and psychotherapy visits account for a much smaller share of total outpatient care. When converted to a per-population basis, the psychotropic visit rate is far higher for older adults, demonstrating the extent to which these medications permeate outpatient office-based care.

Given the financial and time constraints on clinical care and training, these analyses provide data that health systems can use as they shepherd resources in the manner that best serves patient need. The workforce challenges in psychiatry are long-standing, and these findings demonstrate the limited role that psychiatrists play in the delivery of mental health care to older adults. In addition, this work highlights the pervasiveness of psychotropic use in the outpatient care of older adults, heightening concern about the potential appropriateness of use. ${ }^{8,9,36}$ There is tremendous need to develop and implement systems of care that can serve people where they are and where they wish to be seen; ${ }^{15,37}$ for older adults, this means outside of specialty mental health care. It is hoped that new Medicare-led initiatives in bundled payment and populationbased care will make implementation of models such as collaborative care more financially viable. ${ }^{38,39}$ Regardless, it is critical that nonpsychiatric providers receive more training in and support for the management of mental disorders in their clinical practices.

\section{ACKNOWLEDGMENTS}

This work was supported by the Beeson Career Development Award Program (NIA K08AG048321, AFAR, The
John A. Hartford Foundation, and The Atlantic Philanthropies).

Conflict of Interest: The authors have no conflicts to disclose.

Author Contributions: Donovan T. Maust acquired and analyzed the data and prepared the first draft of this manuscript. All authors edited the manuscript, helped interpret the results, and guided additional study questions.

Sponsor's Role: None.

\section{REFERENCES}

1. Institute of Medicine. The Mental Health and Substance Use Workforce for Older Adults: In Whose Hands? Washington, DC: National Academies Press, 2012.

2. Bartels SJ, Naslund JA. The underside of the silver tsunami-older adults and mental health care. N Engl J Med 2013;368:493-4963.

3. Jeste DV, Alexopoulos GS, Bartels SJ et al. Consensus statement on the upcoming crisis in geriatric mental health: Research agenda for the next 2 decades. Arch Gen Psychiatry 1999;56:848-853.

4. Crystal S, Olfson M, Huang C et al. Broadened use of atypical antipsychotics: Safety, effectiveness, and policy challenges. Health Aff (Millwood) 2009;28:w770-w781.

5. Comer JS, Olfson M, Mojtabai R. National trends in child and adolescent psychotropic polypharmacy in office-based practice, 1996-2007. J Am Acad Child Adolesc Psychiatry 2010;49:1001-1010.

6. Schwarz A. Attention Disorder or Not, Children Prescribed Pills to Help in School. The New York Times, 2012 [on-line]. Available at http://www.nytimes.com/2012/10/09/health/attention-disorder-or-not-children-prescribedpills-to-help-in-school.html? pagewanted=all\&_r=0 Accessed March 8, 2014.

7. Schwarz A. Doctors train to spot signs of A.D.H.D. in children. The New York Times, 2014 [on-line]. Available at http://www.nytimes.com/2014/02/ 19/health/doctors-train-to-evaluate-anxiety-cases-in-children.html Accessed March 8, 2014.

8. Maust DT, Oslin DW, Marcus SC. Effect of age on the profile of psychotropic users: Results from the 2010 National Ambulatory Medical Care Survey. J Am Geriatr Soc 2014;62:358-364.

9. Wiechers IR, Kirwin PD, Rosenheck RA. Increased risk among older veterans of prescribing psychotropic medication in the absence of psychiatric diagnoses. Am J Geriatr Psychiatry 2014;22:531-539.

10. Klap R, Unroe KT, Unutzer J. Caring for mental illness in the united states: A focus on older adults. Am J Geriatr Psychiatry 2003;11:517-524.

11. Hoffman C, Rice D, Sung H-Y. Persons with chronic conditions. JAMA 1996;276:1473-1479.

12. Lotrich FE, Pollock BG. Aging and clinical pharmacology: Implications for antidepressants. J Clin Pharmacol 2005;45:1106-1122.

13. Pollock BG. The pharmacokinetic imperative in late-life depression. J Clin Psychopharmacol 2005;25:S19-S23.

14. American Geriatrics Society Beers Criteria Update Expert Panel. American Geriatrics Society updated Beers Criteria for potentially inappropriate medication use in older adults. J Am Geriatr Soc 2012;60:616-631.

15. Gum AM, Areán PA, Hunkeler E et al. Depression treatment preferences in older primary care patients. Gerontologist 2006;46:14-22.

16. Stanley MA, Calleo J, Bush AL et al. The peaceful mind program: A pilot test of a cognitive-behavioral therapy-based intervention for anxious patients with dementia. Am J Geriatr Psychiatry 2013;21:696-708.

17. Cheston R, Jones K, Gilliard J. Group psychotherapy and people with dementia. Aging Ment Health 2003;7:452-461.

18. Bishop TF, Press MJ, Keyhani S et al. Acceptance of insurance by psychiatrists and the implications for access to mental health care. JAMA Psychiatry 2014;71:176-181.

19. American Geriatrics Society. How Many Board Certified Geriatricians and Geropsychiatrists Are There in the US? [on-line]. Available at http:// www.americangeriatrics.org/advocacy_public_policy/gwps/gwps_faqs/id:3183 Accessed April 14, 2014.

20. Olfson M, Blanco C, Wang S et al. National trends in the mental health care of children, adolescents, and adults by office-based physicians. JAMA Psychiatry 2014;71:81-90.

21. National Center for Health Statistics, Centers for Disease Control and Prevention. National Ambulatory Medical Care Survey: 2010 NAMCS Micro-data file documentation [on-line]. Available at ftp://ftp.cdc.gov/pub/Health_Statis- 
tics/NCHS/Dataset_Documentation/NAMCS/doc2010 Accessed December 12, 2013.

22. Hsiao C-J. National Center for Health Statistics, Centers for Disease Control and Prevention. Understanding and Using NAMCS and NHAMCS Data: Data Tools and Basic Programming Techniques [on-line]. Available at http://www.cdc.gov/nchs/ppt/nchs2010/03_Hsiao.pdf Accessed December 28, 2013.

23. U.S. Census Bureau, U.S. Department of Commerce. Population Estimates: Population and Housing Unit Estimates [on-line]. Available at http:// www.census.gov/popest/index.html Accessed December 28, 2013.

24. Gardner MJ, Altman DG. Confidence intervals rather than $\mathrm{p}$ values: Estimation rather than hypothesis testing. BMJ 1986;292:746-750.

25. Olfson M, Marcus SC. National patterns in antidepressant medication treatment. Arch Gen Psychiatry 2009;66:848-856.

26. Olfson M, Marcus SC. National trends in outpatient psychotherapy. Am J Psychiatry 2010;167:1456-1463.

27. Marcus SC, Olfson M. National trends in the treatment for depression from 1998 to 2007. Arch Gen Psychiatry 2010;67:12651273.

28. Mojtabai R, Olfson M. National trends in psychotropic medication polypharmacy in office-based psychiatry. Arch Gen Psychiatry 2010;67:2636.

29. Kane JM, Woerner M, Lieberman J. Tardive dyskinesia: Prevalence, incidence, and risk factors. J Clin Psychopharmacol 1988;8:52S-56S.

30. Jeste DV, Rockwell E, Harris MJ et al. Conventional vs. newer antipsychotics in elderly patients. Am J Geriatr Psychiatry 1999;7:70-76.
31. Field TS, Gurwitz JH, Harrold LR et al. Risk factors for adverse drug events among older adults in the ambulatory setting. J Am Geriatr Soc 2004;52:1349-1354.

32. Howden LM, Meyer JA. U.S. Census Bureau, U.S. Department of Commerce. Age and Sex Composition: 2010 [on-line]. Available at http:// www.census.gov/prod/cen2010/briefs/c2010br-03.pdf Accessed December 28, 2013.

33. Billioti de Gage S, Bégaud B, Bazin F et al. Benzodiazepine use and risk of dementia: Prospective population based study. BMJ 2012;345: e6231.

34. Billioti de Gage S, Moride Y, Ducruet T et al. Benzodiazepine use and risk of Alzheimer's disease: Case-control study. BMJ 2014;349:g5205.

35. Cooper LA, Gonzales JJ, Gallo JJ et al. The acceptability of treatment for depression among African-American, Hispanic, and white primary care patients. Med Care 2003;41:479-489.

36. Mojtabai R. Diagnosing depression in older adults in primary care. N Engl J Med 2014;370:1180-1182.

37. Chen H, Coakley EH, Cheal K et al. Satisfaction with mental health services in older primary care patients. Am J Geriatr Psychiatry 2006;14:371379.

38. Maust DT, Oslin DW, Marcus SC. Mental health care in the accountable care organization. Psychiatr Serv 2013;64:908-910.

39. Katon WJ, Unutzer J. Health reform and the affordable care act: The importance of mental health treatment to achieving the triple aim. J Psychosom Res 2013;74:533-537. 


\section{APPENDIX}

Table A1. Psychotropic Drugs Reported in the 2007-2010 National Ambulatory Medical Care Survey

\begin{tabular}{|c|c|c|c|}
\hline Antidepressants & Anxiolytics & Antipsychotics & Mood Stabilizers \\
\hline Amoxapine & Buspirone & Fluphenazine & Lamotrigine \\
\hline Citalopram & Butalbital & Loxapine & Valproate/divalproex \\
\hline Clomipramine & Chloral hydrate & Molindone & \\
\hline Doxepin & Chlordiazepoxide & Perphenazine & Stimulants \\
\hline Duloxetine & Clonazepam & Pimozide & Methylphenidate \\
\hline Fluvoxamine & Diphenhydramine & Thiothixene & Dextroamphetamine \\
\hline Imipramine & Doxepin & Trifluoperazine & Pemoline \\
\hline Maprotiline & Doxylamine & Second Generation: & Atomoxetine \\
\hline Mirtazapine & Estazolam & Aripiprazole & \\
\hline Nefazodone & Eszopiclone & Clozapine & \\
\hline Nortriptyline & Flurazepam & Olanzapine & \\
\hline Sertraline & Oxazepam & & \\
\hline Tranylcypromine & Pentobarbital & & \\
\hline Trazodone & Phenobarbital & & \\
\hline \multirow[t]{8}{*}{ Venlafaxine } & Prazepam & & \\
\hline & Pyrilamine & & \\
\hline & Ramelteon & & \\
\hline & Temazepam & & \\
\hline & Triazolam & & \\
\hline & Tybamate & & \\
\hline & Zaleplon & & \\
\hline & Zolpidem & & \\
\hline
\end{tabular}

